\title{
Open
}

\section{Report on the Banbury Summit Meeting on medical genetics training in the genomic era, 23-26 February 2014}

\author{
Bruce R. Korf, MD, PhD ${ }^{1}$, Miriam G. Blitzer, $\mathrm{PhD}^{2}$, Laurie A. Demmer, $\mathrm{MD}^{3}$, \\ Gerald L. Feldman, MD, $\mathrm{PhD}^{4}$ and Michael S. Watson, $\mathrm{PhD}^{5}$
}

\section{CURRENT LANDSCAPE OF MEDICAL GENETICS TRAINING}

The ABMG was founded in 1980. From 1984 to 1995, it accredited all medical genetics training programs and was also responsible for administering the certification exams to all graduates of training programs. At the outset, multiple training and accreditation categories were recognized, and certificates offered in clinical genetics, clinical biochemical genetics, clinical cytogenetics, and genetic counseling (Figure 1). Physicians could achieve certification as clinical geneticists, and either physicians or PhDs could achieve certification as clinical laboratory geneticists, with separate training and certification in clinical cytogenetics and clinical biochemical genetics. PhDs could also be certified as medical geneticists. The ABMG administered the certification examinations for genetic counselors through 1993; they did not accredit genetic counseling training programs, but did approve sites for clinical training. In 1990 clinical molecular genetics was approved as a new laboratory specialty; the first certification exam was given as a joint examination with clinical biochemical genetics, and then in 1993 clinical molecular genetics certification was offered as a third distinct laboratory specialty. $\mathrm{PhD}$ medical genetics certification was not offered after 2005 due to a dwindling number of trainees.

The ABMG was accepted by the ABMS as the 24th member specialty board in 1991. Subsequently, the Accreditation Council for Graduate Medical Education (ACGME) assumed responsibility for the accreditation of medical genetics residency training programs by establishing a medical genetics residency review committee. The ABMG continued to be the certifying organization for all medical genetics specialties and continued to accredit medical genetics laboratory training programs. The American Board of Genetic Counseling was incorporated in 1993 and took on the role of independently certifying genetic counselors in 1996. Formal accreditation of genetic counseling graduate programs by this board began in 1995. In 1999, a new subspecialty in molecular genetic pathology, sponsored by the ABMG and the American
Board of Pathology, was approved by the ABMS, with training programs accredited by the ACGME by a review committee comprised members drawn from the medical genetics and pathology residency review committee. A subspecialty in medical biochemical genetics, sponsored by the ABMG, was approved by the ABMS in 2007, with ACGME accreditation under the aegis of the medical genetics residency review committee. The ABMG, with formal approval from the ABMS, changed its name to the ABMGG in 2014.

In the time since the initiation of formal medical genetics training, growth in the number of new medical geneticists has been relatively small, though with variability between specialties over time (Figure 2). This issue was the subject of previous Banbury meetings on medical genetics. ${ }^{1,2}$ Numbers of clinical cytogeneticists have been stable but more and more are becoming dual-certified in both clinical cytogenetics and clinical molecular genetics. The numbers seeking clinical molecular genetics training have grown. Training programs have limited funding, so many applicants have difficulty finding a funded training position. The number of genetic counselors has steadily increased, though not necessarily in proportion to the opportunity to incorporate genetics and genomics into medical practice, perhaps in part due to challenges in billing for genetic counseling services and the expanded role of genetic counselors in industry.

\section{GUIDING PRINCIPLES FOR MEDICAL GENETICS TRAINING}

Participants in the meeting identified a set of guiding principles, which are discussed below.

1. Training of medical geneticists must remain broad-based to encompass the full range of genetic conditions across the life span.

Issues in medical genetics span the full range of medicine, encompassing essentially all body systems and all areas of medical specialty. This has led some to seek joint training in medical genetics and other

${ }^{1}$ Department of Genetics, University of Alabama at Birmingham, Birmingham, Alabama, USA; ${ }^{2}$ Department of Pediatrics, University of Maryland School of Medicine, Baltimore, Maryland, USA; ${ }^{3}$ Levine Children's Specialty Center, Carolinas HealthCare, Charlotte, North Carolina, USA; ${ }^{4}$ Departments of Pediatrics and Pathology, Wayne State University School of Medicine, Detroit, Michigan, USA; ${ }^{5}$ American College of Medical Genetics and Genomics, Bethesda, Maryland, USA. Correspondence: Bruce R. Korf (bkorf@uabmc.edu) 


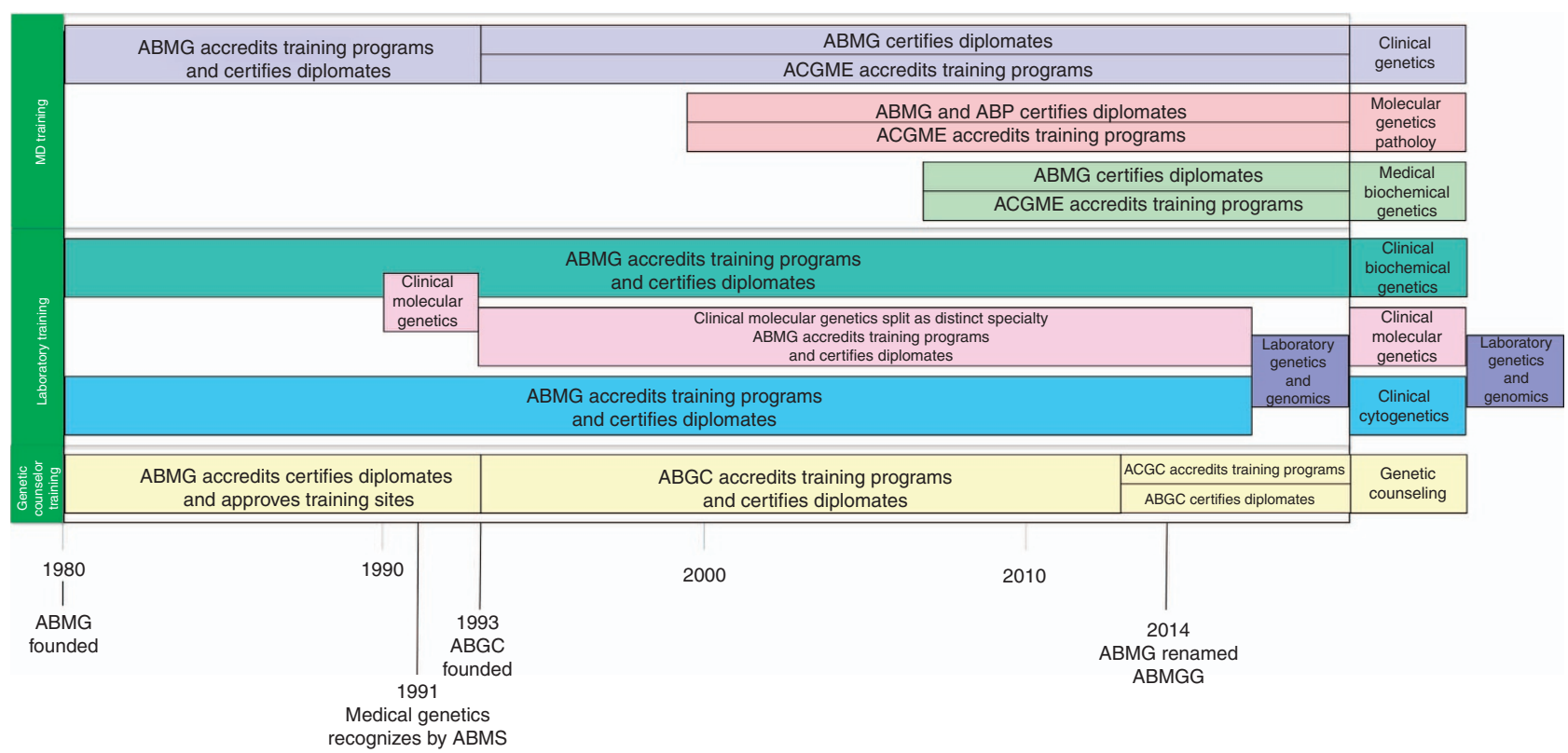

Figure 1 Evolution of medical genetics training. ABGC, American Board of Genetic Counseling; ABMG, American Board of Medical Genetics; ABMGG, American Board of Medical Genetics and Genomics; ABMS, American Board of Medical Specialties; ABP, American Board of Pathology; ACGC, Accreditation Council for Genetic Counseling; ACGME, Accreditation Council for Graduate Medical Education.

specialties, such as neurology or oncology. It has also led to the suggestion that medical genetics training could be included as a fellowship subspecialty to follow training in other specialties. The concern about this notion is that there might be too few subspecialty trainees to sustain individual training programs in areas such as neurogenetics or cancer genetics, and even fewer in areas where genetics and genomics are at an earlier stage of development, yet where there currently exist some approaches that may benefit patients. Furthermore, medical geneticists are trained to address the clinical significance of genetic variants in light of a patient's clinical status, and this skill set requires significant training time beyond that which it may be possible to include in a one-year fellowship. The group's opinion was that, rather than build a large number of genetics subspecialty fellowships, it would be better to ensure that medical geneticists receive broad training and be prepared to partner with colleagues in other medical specialties to help manage the needs of their patients.

2. Clinical geneticists, genetic counselors, and laboratory geneticists must work as an integrated team.

Clinical geneticists, genetic counselors, and laboratory geneticists have complementary skills and their contributions to patient care should be integrated. Clinical geneticists are best equipped to diagnose patients, to help to interpret laboratory results in the context of patient care, and to manage clinical problems, including surveillance for complications of a genetic disorder and, where possible, treatment. Genetic counselors are trained to interpret and explain genetic risks to patients in light of family history, genetic and genomic testing, and the patient's medical history. Laboratory geneticists work with clinicians to formulate the most effective testing strategy, perform the test(s), and provide an interpretation of the results to the ordering clinician. These roles overlap and lend themselves to a team approach; not all members of the team will always be involved in caring for every patient, but the skills of each should be available to meet patient needs as appropriate.

3. The medical genetics team must work collaboratively with other medical specialties. The role of the genetics team will vary according to the needs of the patient. In some cases, the medical geneticist may be in the best position to follow and even treat a patient; for example, if the clinical problem involves multiple systems or an inborn error of metabolism. In other cases, a specific genetic disorder will fit clearly within the scope of practice of another medical specialty; for example, a patient with a genetic cardiomyopathy would be treated by a cardiologist, although the interpretation of genetic testing might be done by the medical genetics team. We would favor including training in basic principles of genetics and genomics in all medical specialties, but recognize the challenge of including sufficient background to achieve competency in this complex area given the other demands on specialist training. If subspecialty fellowships are not to be offered, it will be important for medical geneticists to work together with other medical specialists to be sure that genetic and genomic approaches are appropriately integrated into routine care. 
a

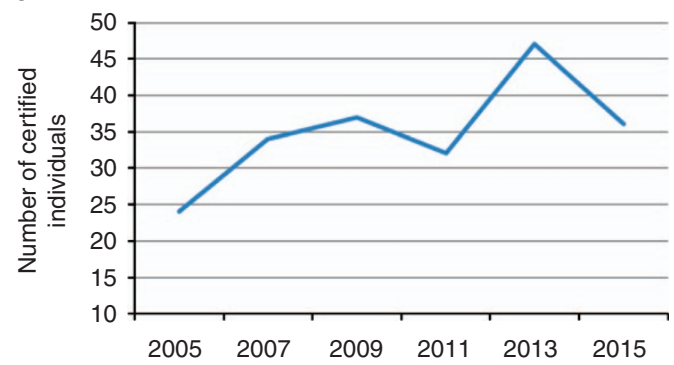

C

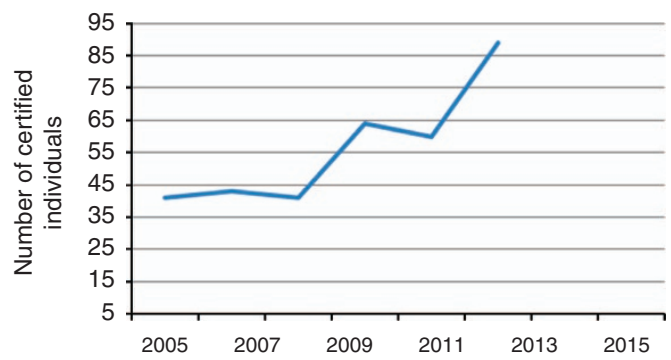

b

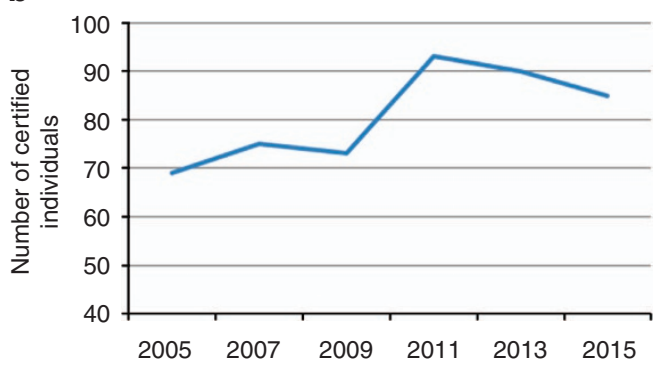

d

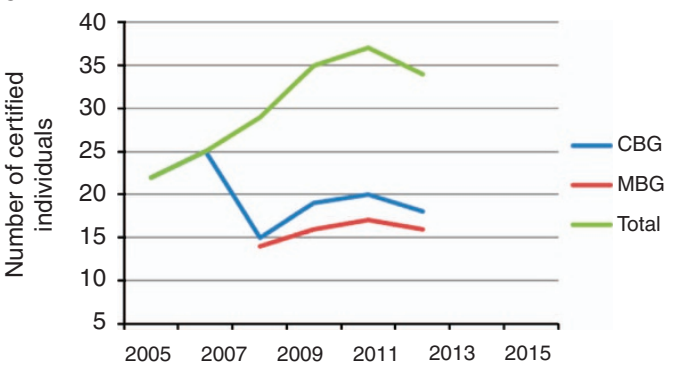

Figure 2 Numbers of certified geneticists in various fields between 2005 and 2015. (a) Clinical cytogeneticists. (b) Clinical geneticists. (c) Clinical molecular geneticists. (d) Biochemical geneticists. CBG, Clinical biochemical geneticists; MBG, Medical biochemical geneticists.

4. Medical genetics training programs must provide broad-based education, which may be facilitated using innovative approaches.

If medical geneticists are going to be able to approach medical problems across the phenotypic spectrum and incorporate genomics into their practice, these broad areas will need to be integrated into their training. This can create a challenge in some programs, especially those that are small, to cover areas that may not be emphasized in clinical practice in that program. There is also a need to develop a cadre of teachers in the new area of genomics, recognizing that this was not an area of training in the previous generation. These challenges may be best addressed through innovative approaches to supplement clinical teaching, including, for example, the use of simulated patients, didactic courses or case conferences that span multiple institutions.

5. Medical genetics training must continually evolve so that trainees are prepared to apply the rapid advances in genomic medicine.

The entire context for this discussion is the need for training to evolve as the field of genetics and genomics evolves. This will be an ongoing process, so the training paradigms will need to continually adapt, which can be a challenge given that medical training is an increasingly regulated and complex enterprise.

6. Medical genetics workforce needs will change with increasing opportunities in genomic medicine and the use of informatics approaches to improve efficiency.

The steady but small numbers of individuals who enter medical genetics training has already been noted.
Although the numbers may be increased by training more genetic counselors, which can be done more quickly and less expensively than training more physician geneticists, some increase in the workforce will be needed as genetics and genomics is integrated across all areas of medicine. Efforts can also be made to improve the efficiency of medical genetic and genomic services, especially using tools of information technology and social networking.

\section{RECOMMENDATIONS FOR MEDICAL GENETICS AND GENOMICS TRAINING}

Given the principles stated above, the attendees at the meeting offered a set of recommendations for the evolution of medical genetics and genomics training.

\section{Integration of genetics and genomics into medical specialties}

As noted above, the meeting participants did not embrace the idea of proliferating genetics subspecialty training into other medical specialties. This concept has been considered for some time, and might be viable in some areas where genetics has been adopted as an important approach, such as oncology, neurology, and cardiology. Indeed, some individuals have customized their own training to obtain expertise in more than one area. In other cases, individuals who were trained in one area of medicine have made the effort to selflearn how to incorporate genetics and genomics into their practice. Nonetheless, we believe that this will not be a sustainable model for all areas of medicine, and instead 
encourage medical geneticists to be broadly trained and to expect to collaborate with colleagues across all medical specialties. This will require that collaboration be incorporated into training, as well as into medical practice. Medical genetics trainees need to have opportunities to interact with colleagues in other areas of medicine, including through participation in multidisciplinary clinical programs and case conferences in various specialties. The use of innovative approaches, as noted previously, can also help fill gaps in specific training programs. Most importantly, trainees need to be able to look to their mentors as role models in interdisciplinary care.

Even as medical geneticists must collaborate with other specialists, there remains a great need to educate nongeneticists on the use of genetics and genomics in their practice. Rather than convince them that they can practice genetics without formal training, this exposure is likely to convince them of the importance of genetics and genomics and of the need to engage experts in helping them to provide care to their patients. The medical genetics community should therefore be encouraged to reach out to other medical specialties to help educate their members about the application of genetics and genomics at all levels of training, including pre-graduate, residency, fellowship, and continuing education.

\section{Genomic medicine}

As previously noted, the application of genomics raises a host of new challenges and opportunities in medical practice, especially since a large proportion of the current training faculty received their own training in the pre-genomic era. This is not a new problem in medicine, as many specialties have had to adapt to new technologies; for example, the advent of new methods of imaging in radiology. Recognizing this, the ABMGG has updated the names of existing training programs to "Clinical Genetics and Genomics," "Clinical Cytogenetics and Genomics," and "Clinical Molecular Genetics and Genomics" (also see below for information about the new Laboratory Genetics and Genomics specialty, a merged specialty that is replacing clinical cytogenetics and clinical molecular genetics programs).

Genomic medicine incorporates competencies that are already part of the training of medical geneticists; for example, distinguishing between variants of unknown significance and pathological variants. Other areas, especially the use of informatics tools to analyze the very large data sets that result from genomic sequencing, have not traditionally been part of medical genetics training. The need to be able to incorporate phenotypic data from other areas of medicine has also been mentioned already. Both needs will benefit from programs to train medical geneticists to practice in new areas through continuing education courses, and perhaps a "genomics academy" to provide intensive training over a relatively short period. The use of shared educational offerings and simulations, also as noted above, can be helpful to create a genomics-competent genetics workforce.

\section{Therapeutics}

With the exception of biochemical genetics, medical geneticists have not, in the past, been heavily involved in the treatment of patients. Most of the rare disorders diagnosed by geneticists have not been amenable to treatment, so the clinical encounter has focused mostly on diagnosis and counseling. In some cases, mainly for multisystem disorders such as neurofibromatosis, medical geneticists have taken responsibility for longitudinal management and surveillance. The opportunities for treatment, however, are now rapidly expanding. As we better understand the pathogenesis of genetic disorders, usually by studyingthe function of the gene product, opportunities are being recognized for intervention. There are many current examples, such as the treatment of tuberous sclerosis complex or cystic fibrosis with either new or repurposed drugs, as well as several new approaches to the treatment of biochemical genetic disorders.

Although some system-specific disorders, such as seizure disorders, will be treated by other medical specialists, medical geneticists can play a key role in managing, and even treating, patients with complex multi-system disorders that do not easily come under the wing of specific specialists. Moreover, medical geneticists can be involved not only in the implementation of new approaches to treatment, but also in the development and execution of clinical trials to create and validate new approaches. Just as exposure to clinical trials has been incorporated into the training of oncologists, it may need to be incorporated into the training of medical geneticists. Training of other members of the care team, including nurses and other professionals, will also be important.

\section{Laboratory genetics training}

Aside from the need to incorporate new technologies in all areas of laboratory genetics training and to include genomic approaches, there is a specific issue regarding clinical cytogenetics training. Cytogenetics was the first of the laboratory genetics specialties. It began in the early 1960s when practical approaches were introduced to study individuals for chromosomal anomalies and the first trisomy and deletion syndromes were delineated (i.e., trisomy 21 in Down syndrome). The field has evolved greatly over the ensuing half century, from the introduction of chromosome banding in the 1970 s to fluorescence in situ hybridization in the 1980s and cytogenomic microarray testing in the 2000s. With the latter, cytogenetics has embraced genomics, and the boundary between cytogenetics and molecular genetics has become blurred. Furthermore, some genetic conditions cannot be diagnosed solely by genomic sequencing, as copy number changes that require cytogenomic approaches account for some cases. Hence, the skills needed to achieve molecular diagnosis include those both of cytogenetics and molecular genetics. This raises the question of whether cytogenetics and molecular genetics should be merged into a single training path. Banbury meeting participants recommended that consideration be given to such a merger, and this was taken 
up by the ABMGG, resulting in the creation of a new specialty: "Laboratory Genetics and Genomics". This new specialty has been approved by the ABMS and consists of clinical laboratory training that combines and integrates experiences with both cytogenetic and molecular genetic approaches. This training path will replace the existing Clinical Cytogenetics and Genomics and Clinical Molecular Genetics and Genomics programs, as of July 2017.

\section{Recruitment and outreach}

Medical genetics may have a recognized place as a distinct medical specialty, but it does not have a well-defined place in the list of careers considered by prospective trainees. The issues for clinical and laboratory training are different. For clinical genetics, there is a paucity of individuals who seek medical genetics residency training (see Figure 2). In part this may be due to lack of recognition by medical students of the possibility of medical genetics training. In part it may reflect a perception that medical genetics deals only with rare untreatable disorders, with little opportunity to intervene. In part it may reflect a recognition that genetic services are timeintensive and poorly reimbursed. As has been noted already, the scope of activities in modern genetics and genomics has expanded beyond just diagnosis and counseling, although this opportunity may not have been communicated well to prospective trainees. Professional societies such as the American College of Medical Genetics and Genomics can provide their members with educational approaches, such as a summer scholars program and medical student interest groups, to help inform students of the opportunities in medical genetics. Solving the reimbursement issues will also be critical to stimulate more students to seek medical genetics training, as is addressed below.

For laboratory genetics, the issues are likely to be more a matter of funding for training. Many training programs have more applicants than funded positions. Unlike medical genetics training, there is no option of using institutional graduate medical education funds to support laboratory training. For the most part this is absorbed by laboratory budgets or training grants, which are increasingly constrained by restricted payment for laboratory genetic testing, as well as competition from commercial laboratories. Indeed, given that commercial laboratories are increasingly providing genetic testing and require trained personnel, some method for funding training in academic environments should be sought to maintain the flow of qualified laboratory personnel.

\section{Economic issues}

The complexity of medical genetic and genomic testing and care makes them expensive, and in a health care system increasingly concerned about ballooning costs, the argument to incorporate these new technologies faces an uphill climb. There is a need to document the economic value of establishing a diagnosis using a genomic approach, even if the diagnosis only reduces the need for further testing and diagnostic evaluation and does not result in a treatment that changes outcomes. In addition, the medical genetics community should work to reduce costs, not only of genomic sequencing, which is occurring already, but also of interpretation and counseling. Finally, there may be new economic paradigms that involve collaboration of commercial, academic, private, and government partners who think of healthcare on a population-wide basis rather than one patient at a time for conditions for which genomic approaches may prove to be cost effective in maintaining health and preventing disease.

\section{CONCLUSION}

The opportunities to improve health through better approaches to prevention, diagnosis, and treatment using the application of genetic and genomic approaches have never been greater. Realizing these opportunities, however, will require a prepared workforce, as well as a receptive public and payers. Genetics and genomics will affect the practice of medicine in all areas of specialty, yet we believe strongly that there remains a place for broadly trained clinical and laboratory geneticists who embrace the new technologies of today and others yet to come. We hope that the suggestions provided in this document will stimulate the community to evolve its training approaches, and engender enthusiasm within prospective trainees for theirparticipation in genomic medicine both in the clinic and in the laboratory.

\section{ACKNOWLEDGMENTS}

We thank the American College of Medical Genetics and Genomics for its support of this meeting.

\section{DISCLOSURE}

B.R.K. is a member of the Accolade, Envision Genomics and Genome Medical advisory boards; was a recipient of grants from Novartis, the Department of Defense and $\mathrm{NIH}$; and has undertaken educational activities with Quantia and Axis. The other authors declare no conflict of interest.

\section{REFERENCES}

1. Korf BR, Feldman G, Wiesner GL. Report of Banbury Summit meeting on training of physicians in medical genetics, October 20-22, 2004. Genet Med 2004; 7:433-438.

2. Korf BR, Ledbetter D, Murray MF. Report of the Banbury Summit Meeting on the evolving role of the medical geneticist, February 12-14, 2006. Genet Med 2008;10:502-507.

\section{This work is licensed under a Creative Commons} Attribution-NonCommercial-NoDerivs $\quad 4.0$ International License. The images or other third party material in this article are included in the article's Creative Commons license, unless indicated otherwise in the credit line; if the material is not included under the Creative Commons license, users will need to obtain permission from the license holder to reproduce the material. To view a copy of this license, visit http://creativecommons.org/licenses/ by-nc-nd/4.0/

(c) The Author(s) 2017 


\author{
APPENDIX \\ Appendix Meeting Participants \\ Meeting Organizers: Bruce R. Korf, MD, PhD (Chair), \\ University of Alabama at Birmingham; Miriam Blitzer, $\mathrm{PhD}$ \\ (Co-chair), University of Maryland; Laurie Demmer, MD \\ (Co-chair), Carolinas HealthCare System; Gerald Feldman, \\ $\mathrm{MD}, \mathrm{PhD}$ (Co-chair), Wayne State University; Michael \\ Watson, $\mathrm{PhD}$ (Co-chair), American College of Medical \\ Genetics and Genomics. Participants: Robin Bennett, MS, \\ University of Washington; Sarah Bowdin, MD (Sick Kids \\ Toronto); Wendy Chung, $\mathrm{MD}, \mathrm{PhD}$, Columbia University; \\ Julie Gastier-Foster, PhD, Nationwide Children's Hospital \\ and Ohio State University; Susan Hahn, MS, University of \\ Miami; Gail Herman, MD, PhD, Ohio State University; Fuki
}

Hisama, MD, University of Washington; Jennifer Hoskovec, MS, University of Texas; Anne Matthews, RN, PhD, Case Western Reserve University; Ian Krantz, MD, The Children's Hospital of Philadelphia and the Perelman School of Medicine at the University of Pennsylvania; Cynthia Morton, $\mathrm{PhD}$, Brigham and Women's Hospital and Harvard Medical School; Kathleen Rao, $\mathrm{PhD}^{1}$, University of North Carolina; Amy Roberts, MD, Harvard Medical School; Nancy C. Rose, MD, Intermountain Healthcare/University of Utah; Christian Schaaf, MD, PhD, Baylor College of Medicine; Alpa Sidhu, $\mathrm{MD}, \mathrm{PhD}$, Wayne State University ${ }^{2}$; Reid Sutton, MD, PhD, Baylor College of Medicine.

${ }^{1}$ Deceased.

${ }^{2}$ Current affiliation: Michigan State University. 